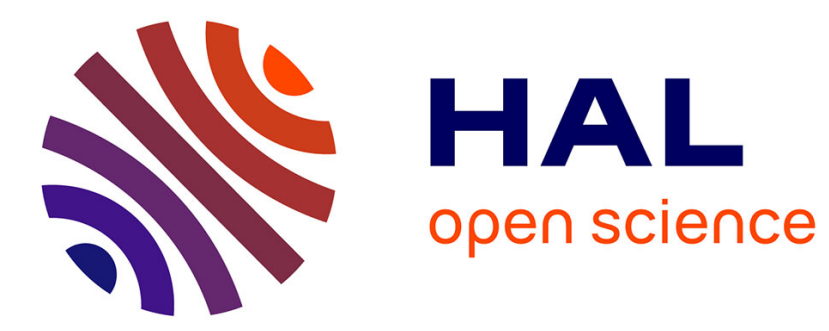

\title{
LE MOUVEMENT DES INTERMITTENTS ET LA CRISE DE LA POLITIQUE CULTURELLE
}

\author{
François Ribac
}

\section{To cite this version:}

François Ribac. LE MOUVEMENT DES INTERMITTENTS ET LA CRISE DE LA POLITIQUE CULTURELLE. Mouvements: des idées et des luttes, 2003, 2003/5 (30), pp.108 - 115 10.3917/mouv.030.0108 . hal-01327301

\section{HAL Id: hal-01327301 https://hal.science/hal-01327301}

Submitted on 6 Jun 2016

HAL is a multi-disciplinary open access archive for the deposit and dissemination of scientific research documents, whether they are published or not. The documents may come from teaching and research institutions in France or abroad, or from public or private research centers.
L'archive ouverte pluridisciplinaire HAL, est destinée au dépôt et à la diffusion de documents scientifiques de niveau recherche, publiés ou non, émanant des établissements d'enseignement et de recherche français ou étrangers, des laboratoires publics ou privés. 


\title{
LE MOUVEMENT DES INTERMITTENTS ET LA CRISE DE LA POLITIQUE CULTURELLE
}

\author{
François Ribac
}

La Découverte | « Mouvements »

2003/5 n³0 | pages 108 à 115

ISSN 1291-6412

ISBN 2-7071-4169-0

Article disponible en ligne à l'adresse :

http://www.cairn.info/revue-mouvements-2003-5-page-108.htm

\section{!Pour citer cet article :}

François Ribac, «Le mouvement des intermittents et la crise de la politique culturelle », Mouvements 2003/5 ( $\left.\mathrm{n}^{\mathrm{o}} 30\right)$, p. 108-115.

DOI 10.3917/mouv.030.0108

Distribution électronique Cairn.info pour La Découverte.

(C) La Découverte. Tous droits réservés pour tous pays.

La reproduction ou représentation de cet article, notamment par photocopie, n'est autorisée que dans les limites des conditions générales d'utilisation du site ou, le cas échéant, des conditions générales de la licence souscrite par votre établissement. Toute autre reproduction ou représentation, en tout ou partie, sous quelque forme et de quelque manière que ce soit, est interdite sauf accord préalable et écrit de l'éditeur, en dehors des cas prévus par la législation en vigueur en France. Il est précisé que son stockage dans une base de données est également interdit. 


\section{Le mouvement des intermittents et la crise de la politique culturelle}

\section{PAR}

\section{FRANÇOIS RIBAC*}

\begin{abstract}
Après l'annulation de nombreux festivals d'été, le mouvement des intermittents semble ne pas faiblir. La liste des initiatives et actions entreprises est longue et la mobilisation a été importante et multiforme. Moins spectaculaires, parce que moins médiatisés, les débrayages, les prises de position de personnes ou d'organisations et les annulations de tournages dans l'audiovisuel ont également été significatifs.
\end{abstract}

$\mathrm{E}$ n donnant son agrément au protocole du 26 juin, le gouvernement Raffarin a montré qu'il en approuvait pleinement les termes et a réitéré la " méthode " qu'on lui connaît dorénavant depuis plusieurs mois. Dans ce cas précis, il a délégué à deux partenaires une décision politique majeure et n'est intervenu dans le conflit qu'à contre-cour. En laissant entendre que le régime était condamné par le MEDEF, le gouvernement a présenté, selon un schéma qui rappelle la récente réforme des retraites, l'accord conclu entre la CFDT et le MEDEF comme un sauvetage inespéré, à prendre ou à laisser.

\section{- Retour rapide sur le régime}

La particularité du système de l'intermittence est de permettre aux allocataires dépendant des annexes 8 et 10 du régime d'indemnisation du chômage, d'être pris en charge entre leurs périodes de travail. Ce système est lié à la réalité de l'emploi dans un secteur où les salariés vont d'un projet à l'autre et sont généralement appelés à travailler pour différents employeurs. De fait il constitue une sorte de financement bis des arts du spectacle et de la production audiovisuelle. Ce régime particulier permet aux compagnies et aux entreprises fragiles de n'embau- cher que temporairement des salarié-e-s, tout en donnant la possibilité aux équipes de préparer les projets.

Le nouveau protocole oblige désormais les salariés à effectuer en dix mois (avec une période transitoire en 2004), au lieu de douze, le quota nécessaire d'heures pour prétendre à une prise en charge. La durée d'indemnisation est réduite de douze à huit mois. Selon La CGT du spectacle et les coordinations, le protocole (malgré quelques replâtrages durant l'été) aura pour résultat de débarquer $30 \%$ des ayantsdroits, soit plusieurs dizaines de milliers de personnes. 15000 ? 20000 ? Plus ? Il n'est guère possible de le déterminer puisque l'UNEDIC n'a jamais fourni le chiffre exact des allocataires. Les estimations varient de 50000 à 100000 selon les sources. De plus, l'accord est tellement complexe qu'il est difficile d'extrapoler la façon dont les Assedic les interpréteront.

Il est évident que la diminution du nombre d'allocataires va fragiliser nombre de compagnies et de structures de production et porter un coup certain à la diversité culturelle dans ces

\footnotetext{
* compositeur
} 
secteurs. La concurrence entre professionnels étant exacerbée, cela devrait également avoir comme conséquence de faire baisser le coût du travail pour les entreprises et d'augmenter la précarisation des salariés. De ce point de vue l'inspiration du projet est clairement libérale et s'inscrit dans "l'air du temps ".

\section{- Un accord politique}

Le protocole, signé par la CFDT et le MEDEF, confirme l'alliance stratégique entre les deux organisations. L'accord a été signé dans une telle précipitation qu'il a même obligé la CFDT à écrire à l'UNEDIC pour lui demander des éclaircissements. Constatant les failles du texte, les partenaires ont dû remanier en catimini le texte final et c'est une version légèrement amputée que le gouvernement a donc agréé. Les recours en justice aboutiront donc probablement à une dénonciation de l'accord.

Comme dans l'affaire des retraites, les conditions rocambolesques dans lesquelles la CFDT a brisé le front syndical soulignent, à rebours, la responsabilité de la gauche plurielle. Durant ses cinq années au pouvoir, le gouvernement Jospin n'a en effet pas jugé bon de réviser les procédures qui permettent de valider des accords signés par des organisations minoritaires avec le patronat. Or, il se trouve que justement la CFDT-spectacle est effectivement minoritaire et que le syndicat majoritaire, la CGT (et FO) ont refusé de signer.

Avec cet accord, ni le MEDEF, ni le gouvernement ne souhaitaient discuter des finalités d'une politique culturelle. Ils ont d'ailleurs centré leur communication sur les " abus " des allocataires (en minorant les dérives avérées dans l'audiovisuel), sur le déficit des annexes et sur la nécessité de "sauver "le régime. Pour le patronat, Jean-Jacques Aillagon et certains décideurs culturels, le problème se réduit à un " trop plein ". Il s'agit simplement de dégraisser (selon l'expression d'un ministre socialiste de l'éducation) le régime de l'intermittence et d'en éliminer " les médiocres ". Jean-Jacques Aillagon avait d'ailleurs publiquement exprimé son sentiment qu'il y avait " trop de compagnies ". À cet endroit du raisonnement, on n'est pas exactement dans la vulgate libérale, mais plutôt dans "l'excellence ", un registre dont les partisans se recrutent aussi bien à droite qu'à gauche. On a beaucoup entendu cet été les patrons des festivals relayer ce genre d'arguments avant d'utiliser, pour contrer les assemblées générales, l'argument des retombées économiques.

En réalité, il est désormais de notoriété publique que les "détournements " sont particulièrement patents dans l'audiovisuel et tout particulièrement du côté des chaînes nationales de télévision publiques et privées. À part une vague commission et des déclarations de principe, le ministre n'a rien engagé pour remédier à ce problème. Probablement parce que le travail d'un ministre de la communication consiste à ne surtout pas importuner TF1. Il faut noter qu'au moment même où le gouvernement agréait le protocole, il défendait mordicus l'exception culturelle à Bruxelles, c'est-à-dire les industries nationales de la culture. Il y aurait également beaucoup à dire de ce côté.

\section{- Une rentrée agitée}

À la rentrée, le SYNDEAC, Syndicat national des entreprises artistiques et culturelles, qui n'est pas affilié au MEDEF, a refusé de participer aux assises régionales de la culture proposées en catastrophe, par Jean-Jacques Aillagon, ce dernier n'ayant pas hésité à recourir aux pressions et aux menaces. Comme cette organisation, la Fedurock (scènes de musiques actuelles) et de nombreuses associations ou groupements professionnels se sont ralliés à la position de la CGT, de FO et de la trentaine de coordinations régionales d'intermittents. Ils ont demandé, comme préalable à toute discussion, un moratoire ou l'abrogation du protocole. En septembre, un certain nombre de structures de diffusion ont même annoncé leur intention de geler régulièrement leurs activités durant l'année ou même d'annuler leur saison. Au risque de se ridiculiser, le ministre a donc été contraint d'annuler les assises en région. Pour la première fois depuis 1968, le divorce entre le ministère de la Culture et les milieux artistiques 
semble total. Ce fait est d'importance, dans la mesure où les politiques menées par les ministres de droite ou de gauche se situent, depuis les débuts de la politique Lang, dans une relative continuité. Ce consensus a hélas été confirmé par l'échec cuisant de Catherine Trautman, qui avait tenté d'élaborer une charte publique du spectacle vivant et défendu la techno contre la criminalisation. Le ministre est conscient de ce problème et c'est sans doute pourquoi il a chargé un ancien conseiller de François Miterrrand de préparer un débat national pour janvier 2004.

\section{- L'éclatement de la « grande famille des artistes ॥}

La crise a révélé les profondes disparités dans les différents mondes de l'art. Le débat sur les formes d'action a montré qu'en fonction de la notoriété et de la situation acquise, de l'histoire de chacun-e, de la profession et de la discipline que l'on pratique, les intérêts et les attitudes étaient différents. Aux disparités au sein d'un même domaine (cinéma ou théâtre par exemple), s'ajoute une prise de conscience quant aux limites du régime de l'intermittence. On s'est ainsi rendu compte qu'un certain nombre de métiers (par exemple les auteurs) ou de disciplines (arts plastiques, musiques populaires, jazz) ne bénéficiaient pas d'une protection sociale élémentaire. Le régime de l'intermittence est surtout adapté aux professions du spectacle et aux industries de l'audiovisuel et n'empêche pas l'inégalité et l'exclusion de se développer.

Mais alors que la dynamique solidaire concourait à rapprocher les différentes disciplines, les déchirements ont été particulièrement forts (et médiatisés) dans les arts du spectacle. Il en a résulté une véritable rupture symbolique, à forte dimension générationnelle. Tout au long de l'été, cette fêlure s'est accentuée. Lors du festival d'Aix en Provence, perturbé par des manifestations et finalement annulé, on a ainsi pu entendre Jérôme Deschamps contester à des " jongleurs " le droit de bénéficier du régime de l'intermittence ou le chef d'orchestre Marc Min- kowski traiter les manifestants de " nazis culturels ". Les prises de positions de deux metteur-e$s$ en scène ont tout particulièrement retenu l'attention. L'un pour dénier au mouvement sa légitimité et soutenir le protocole d'accord (Patrice Chéreau), l'autre, plus nuancée, pour exprimer sa réticence envers le principe d'une grève des spectacles (Ariane Mnouchkine). Audelà de l'indignation que ces deux " entrepreneurs de morale " ont déclenché, leurs réactions posent des questions fondamentales quant à la spécificité de l'activité artistique d'une part et à son évaluation d'autre part. Pour Ariane Mnouchkine, la rupture est d'autant plus imprévue qu'elle avait régulièrement fait du Théâtre du Soleil un lieu d'action politique et humanitaire. Pendant la guerre de Bosnie, elle avait ainsi organisé avec d'autres artistes une grève de la faim pour réclamer la fin de l'agression nationaliste serbe et la levée du siège de Sarajevo. La pièce présentée à Avignon par le Théâtre du Soleil ne dérogeait pas à cette règle puisque la troupe présentait un travail ayant comme thème l'errance des réfugiés. En se demandant si l'annulation des spectacles ne consistait pas à " se tirer une balle dans le pied ", alors même qu'elle avait exposé son corps aux privations de la grève de la faim, elle posait, quasi explicitement, le caractère quasi sacré d'une représentation. Le soubassement d'une telle prise de position est que l'activité artistique n'est pas un travail comme les autres mais une sorte de moment de vérité ultime dans lequel il est impensable de débrayer. À l'activité banalisée (désacralisée ?) du travail " ordinaire ", Mnouchkine semble opposer la mission de l'artiste et la singularité de la représentation théâtrale. On reconnaît ici sans peine le débat entre critique artiste et critique sociale. On sait que pour Luc Boltanski et Ève Chiappello, l'opposition frontale entre ces deux registres est une des raisons de la paralysie de la gauche française ${ }^{1}$.

De son côté, la réaction de Chéreau se rapproche des "grands décideurs culturels " évo-

1. L. Boltanski et 亡̀. Chiapello Le nouvel esprit $d u$ capitalisme, Gallimard, 1999. 
qués plus haut. On pourrait résumer sa position par " trop d'intermittents tue la culture ". Mais cela amène à lui poser la question de l'évaluation de la qualité artistique. Qui peut juger de l'excellence d'un spectacle ou d'un artiste? Qui sont les experts et quel est leur mandat? Même si les génies existent (ce que les réévaluations permanentes des historiens des arts démentent), une société démocratique doit-elle édifier une méritocratie culturelle et reléguer les moins bons (ou présumés tels) et les amateurs dans les marges ? Comment saiton qu'un grand est devenu petit? Avignon avec son off paupérisé et son in de plus en plus hermétique est-il un modèle valide?

\section{- Le silence}

\section{des autres " grands "}

Force est de constater que durant les semaines qui ont précédé l'accord du 26 juin, le silence des dirigeants de festivals, de théâtres nationaux, d'institutions, de scènes nationales, de centres chorégraphiques a été assourdissant. À compter de l'annulation d'Avignon, votée par le in, la tendance s'est inversée. C'est la force et la longévité du mouvement qui semble avoir contraint le reste de la "profession " à élaborer une position commune et à vaincre les divisions internes entre partisans, opposants résignés et adversaires décidés de la réforme. On peut également penser que l'élargissement du débat à toute la politique culturelle, que l'on doit aux grévistes d'Avignon, a permis d'ouvrir un espace commun de réflexion et de lutte.

Il est vrai que le SYNDEAC réclame, depuis longtemps, une remise à plat de la politique culturelle et selon son expression un "Valois de la Culture "en allusion aux négociations de Grenelle. Cette position a le mérite de poser la nécessité d'un débat général. Mais elle comporte également des inconvénients. D’une part, le "Valois de la culture " n'est pas sans ambi- guité car il peut signifier un tête à tête entre les organisations représentatives et l'état, en oubliant la nécessité d'un débat préalable. D'autre part, il laisse à l'écart des acteurs essentiels, pourtant convoqués à tout bout de champ, pour justifier les politiques. Enfin, il restreint l'espace symbolique et sociétal de la culture.

Depuis bien longtemps la discussion sur la politique culturelle est trop cantonnée à une négociation sur les moyens et les équipements. Bien que la validité de ces demandes ne soit pas contestable, ces revendications sont plus le fait de responsables qui défendent des outils que d'une réflexion d'ensemble.

Le spectacle vivant (qu'il vaudrait mieux appeler arts du spectacle tant il est vrai qu'on ne sait ce que sont des arts morts) est assimilé, dans un raccourci jamais explicité, à la culture. Cela revient à considérer l'offre de spectacles, les équipements et les équipes qui les animent, comme les acteurs centraux d'une politique publique et plus généralement l'activité professionnelle comme la condition de la qualité artistique. Même s'il ne s'agit pas ici de déconsidérer le secteur et les professionnels, il n'en reste pas moins que cette direction mène directement dans un cul-de-sac.

Notamment parce que l'on fait l'impasse sur la pratique amateur et sur sa place réelle. La rhétorique libérale et le management sont littéralement obsédés par le professionnalisme et ce mouvement résulte du mouvement de marchandisation croissante des activités humaines auxquelles les arts du spectacle n'échappent pas. Quand on sait que la politique des vingt dernières années a précisément constitué à créer des équipements partout et à insister sur les " gisements économiques " que représentaient les activités culturelles, on ne doit pas écarter cette objection. Il n'y a pas d'équivalence entre le professionnalisme et l'art. 
Si l'on prend l'exemple du rock ou du rap, l'activité collective se construit généralement sur la base du bénévolat. Dans ces mondes le professionnel est même regardé avec méfiance. Autre exemple, personne ne considère que Kafka ou le peintre Chaissac ne sont pas des artistes alors même qu'ils n'ont jamais été des " pros ".

Ensuite, c'est faire preuve de cécité historique. Si l'on s'en tient au seul théâtre, un examen attentif montrerait à coup sûr que les animateurs de la décentralisation théâtrale et les plus prestigieux (considérés comme tels) metteurs en scènes actuels sont, dans leur immense majorité, issus de troupes amateurs, du théâtre universitaire, des réseaux d'animation type Léo Lagrange, des MJC, etc.

L'amateur est donc un passionné doté de compétences et d'une érudition, acteur essentiel du monde de l'art où il officie. Pas un dilettante inculte à qui il manquerait quelque chose. Qu'il soit ou non en cours de professionnalisation, ce qui ne saurait être la seule finalité de l'art, il lui arrive d'être le porte-parole de nouvelles formes et d'autres valeurs ${ }^{2}$.

L'exemple de la danse contemporaine ou des musiciens baroques en France, apparus au début des années 1980, confirme un autre fait ; les institutions "en dur " ne constituent en aucun cas une garantie de qualité artistique ou d'innovation, parfois elles sont même le principal frein à des changements esthétiques. Même lorsqu'elles sont disposées à accueillir des "nouveaux" (pourquoi en douter ?), il arrive souvent que leurs équipements et leurs techniques soient inadaptées aux nouveaux langages qui surgissent. Les deux secteurs se sont d'abord développés dans les marges. La reconnaissance du public et de la critique a précédé celle de l'État, longtemps indifférent, et elle s'est faite avec l'hostilité des " pros " du secteur. Les opposants les plus résolus au protocole du 26 juin soulignent, à juste titre, que le régime de l'intermittence assure partiellement l'émergence des nouveaux styles et constitue une sorte de passerelle entre nouveaux et anciens. Il protège, dans une certaine mesure, de l'académisme. On peut craindre qu'avec le nouveau protocole la prochaine danse contemporaine ne puisse plus émerger même si, pour sûr, elle existera. C'est ce que savent les équipes du théâtre de Montpellier ou de Nantes lorsqu'elles mettent leur équipement à la disposition des intermittents en grève et de tous ceux et celles qui se sont prononcés contre le protocole.

\section{- Éloge des luttes}

L'apparition des coordinations a eu le mérite de mettre en cause le statu quo et de braquer les projecteurs sur d'autres protagonistes aussi décisifs (mais pas plus) que les décideurs culturels, les " grands ", la presse, le MEDEF, la CFDT ou le ministre. Significativement, les manifestations d'intermittents ont appelé le public à se joindre à leur lutte. Le mouvement a montré qu'il était doté d'une très forte combativité, d'un sens réel de l'innovation dans les luttes et d'une conscience aiguë des enjeux généraux de notre société ${ }^{3}$. Il ne s'est pas cantonné dans le refus et, en partenariat avec d'autres organisations, a proposé des alternatives à la "réforme ". Par ailleurs, il s'est rapidement tourné vers les autres mouvements sociaux du printemps. La participation d'intermittents au rassemblement du Larzac a ainsi été massive. À l'heure actuelle, le mouvement continue à bénéficier d'un capital de sympathie dans la population, même si les annulations des festivals semblent avoir été mal comprises ${ }^{4}$.

Cette capacité à s'auto-organiser et cette jonction avec les autres mouvements sociaux a probablement surpris les théoriciens qui nous présentaient les mondes artistiques comme les laboratoires du libéralisme et les artistes comme les fourriers du capitalisme. C'était

\footnotetext{
2. A. Hennion, S. Maisonneuve et E. Gomart, Figures de l'amateur, formes, objets, pratiques de l'amour de la musique aujourd'hui, La documentation Française, 2000. 3. Significativement la Coordination d'Ile de France s'appelle coordination des précaires et intermittents.

4. Mi-septembre, un sondage montrait que 61\% des Français sympathisaient avec le mouvement.
} 
oublier que toute crise sociale, et celle-ci est sévère et profonde, est porteuse de possibles et d'une dynamique de dépassement des atonies.

\section{- Convoquer tous les experts (les réseaux)}

En limitant le débat aux organisations professionnelles et à l'état, on confine les usagers des équipements culturels dans le rôle du public, celui même pour lequel on se bat mais sans l'envisager comme un protagoniste actif. Ici, on retrouve un sentiment diffus dans les milieux culturels, que l'intervention des publics pourrait favoriser une politique du " divertissement " et les dérives commerciales : du Puy du Fou à Fort Boyard. L'inconvénient, c'est qu'avec une telle logique, on fait l'égalité entre la culture de masse et les masses. Or cette idée que l'usager est au pire un beauf et au mieux un consommateur nous parle plutôt de la condition de ceux qui le supposent que de la réalité de la société.

Les décennies 1980/1990 ont en effet été marquées par l'irruption d'associations ou d'initiatives d'usagers qui, en s'imposant dans le champ qui les concernait, ont profondément renouvelé les relations entre l'État, les spécialistes, le secteur privé et même les médias. Il suffit par exemple de citer dans le domaine de la santé, Act up, Aides et même le Téléthon ${ }^{5}$. Or ce qui est vrai pour un malade du sida est également vrai pour un spectateur. Ce dernier sait ce qu'il aime et a accumulé une expérience vécue et l'érudition qui l'accompagne. Sa compétence n'est ni inférieure, ni supérieure à celle d'un artiste ou d'un expert ministériel (les fameux inspecteurs de la musique, du théâtre, etc.), elle est la sienne ${ }^{6}$. Il (ou elle) n'a pas besoin d'être sensibilisé (comme si le public était insensible!) comme les politiques publiques s'acharnent à le préconiser. Cette compétence est d'ailleurs prise en compte puisqu'elle est mentionnée dans les brochures de saisons des théâtres, les dossiers de presse et les rapports ministériels. C'est en son nom et en fonction de sa fréquentation (massive ou rare), donc de son expertise, que les réputations et les politiques se construisent et qu'elles sont justifiées. Le mouvement altermondialiste réclame ainsi le partage des décisions et conteste aux seuls experts, par exemple dans les cas des OGM, des scientifiques et des trusts pharmaceutiques, la capacité de décider pour la société. Bien entendu, il ne s'agit pas de dire que les cultureux ou les responsables de structures culturelles se conduisent comme des trusts pharmaceutiques! Simplement il paraît aujourd'hui évident qu'une politique publique de la culture ne peut pas impliquer que les seuls "spécialistes ". On peut même renverser les termes et dire qu'il s'agit de mettre en chantier une discussion où tous les spécialistes interviennent.

Si, comme nous le disent les thuriféraires de la culture, l'art apprend à penser, il est difficile de refuser à tous ceux et celles qui vivent la culture, d'entrer dans le débat. Faute de quoi, on pourrait soupçonner que les bénéfices (intellectuels et sensuels) supposés de la culture sont factices... Or, notre société ne supporte plus, Jean Marie Messier ou l'OMC l'ont appris à leurs dépens, les faux bilans.

On peut ajouter que les métiers de l'art ont besoin de moralisation. Là encore, d'autres secteurs de la société semblent se situer devant nous. Alors qu'en politique le non-cumul des mandats et la séparation entre experts et déci-

\footnotetext{
5. M. Callon, "Ni intellectuel engagé, ni intellectuel dégagé : la double stratégie de l'attachement et du détachement ", in Sociologie du Travail $\mathrm{n}^{\circ}$ 1, vol. 41, 1999, p. 65-78 1999. 6. J.M. Leveratto, La mesure de l'art. Sociologie de la qualité artistique, La Dispute, 2000.
} 
deurs s'imposent progressivement, ces règles devraient être adoptées dans l'estimation de la qualité artistique et des politiques globales. Il n'est ainsi plus possible, notamment en région, que des décideurs soient juges et parties. Il n'est plus possible de voir le cumul et le pantouflage des experts se multiplier. Si l'on dénonce, et avec raison, les effets pervers de la décentralisation Raffarin, on doit rapidement contester les actuelles baronnies, élaborer des règles déontologiques, fixer des objectifs aux structures et organiser la rotation des responsables. Ces principes relèvent plus de l'éthique personnelle des décideurs ou de l'histoire des lieux. Enfin, les milieux de l'art ont besoin de parité, là, comme ailleurs, les hommes continuent à régner sans partage.

\section{- Attention à la fétichisation des artistes}

Dans ce débat, il faudrait veiller à ne pas considérer les techniciens du spectacles et les " intermédiaires " comme de simples parasites, comme l'a récemment fait un compositeur dans une tribune libre à Libération. Ils sont aussi des acteurs essentiels de l'art. Pourquoi ? Mais parce que nous travaillons tous avec des administrateurs(trices), des attaché-e-s de presse et des tourneurs. De plus, sans le travail de l'ingénieur du son de l'IRCAM, le compositeur ne peut pas faire entendre sa musique correctement. La preuve en est que nous parlons toutes et tous (cinéma, théâtre, arts plastiques, musique etc.) de production pour désigner une œuvre. Comme l'a récemment montré Ève Chiapello, cette opposition entre artistes et managers est d'ailleurs devenue factice ou, tout du moins, en voie d'extinction ${ }^{7}$. De toute façon, il semble bien hasardeux de défendre la professionnalisation de l'art et de contester, au même instant, l'apparition d'intermédiaires. Le compositeur oublie un fait historique. C'est la musique savante qui a inventé l'éditeur (un sacré intermédiaire) ! Mieux, la composition classique repose sur le principe d'une exécution par d'autres. Simon Frith a même écrit, et avec raison, que le compositeur et l'éditeur de musique sont les premières personnes qui peuvent vivre de la musique sans l'interpréter ${ }^{8}$. Par conséquent, longue vie aux intermédiaires et aux techniques ${ }^{9}$ !

Encore une fois, ces constats, qui peuvent paraître sévères, ne visent pas à déconsidérer les organisations professionnelles et celles et ceux qui les animent. La politique culturelle a non seulement besoin d'un débat (sous la forme de contre-assises nationales par exemple) mais également que la discussion évite deux écueils :

Le débat ne peut pas concerner les seuls arts du spectacle et oublier les autres disciplines : audio-visuel, arts plastiques, littérature, rock, techno. On doit analyser les raisons pour lesquels la politique culturelle néglige certaines activités, soit en les oubliant, soit en les finançant moins, alors même que depuis 20 ans les ministres (même Aillagon y est contraint) s'affichent dans les techno-parade, lisent de la BD, écoutent les Rolling Stones sur leur auto-radio, se rendent à des expositions de graphes hiphop ou dans des squats d'artistes. Les ministres et leurs conseillers en communication prennent bien en compte les experts et les amateurs (les connaisseurs) de toutes les activités. Mais le problème c'est qu'ils ne justifient pas réellement la disparité de traitement, tant financière que

\footnotetext{
7. È. Chiapello, Artistes versus managers, le management culturel face à la critique artiste, Métailié, 1998.

8. S. Frith, "The popular music industry ", p. 26 à 52 in The Cambridge Companion to pop E Rock, Cambridge, 2001.

9. H.S. BECKER, Les mondes de l'art, Flammarion, 1988.
} 
symbolique, entre disciplines. La seule chose que l'on entend dans les couloirs des DRAC (Directions régionales des affaires culturelles), c'est que " pour donner à Pierre il faudrait prendre à Paul ". Or, ce raisonnement est justement ce avec quoi il faut rompre parce qu'il n'envisage la politique culturelle qu'en termes de masses d'argent et oublie de poser la question des besoins. Dans la mesure où le budget de la culture n'augmente plus (voire régresse) la question est totalement d'actualité.

La culture ne peut être la seule affaire des "professionnels". Elle doit, sous peine d'une nouvelle faillite, impliquer tous les acteurs du secteur et mettre au jour les intérêts (voire les besoins) divergents : publics, programmateurs, presse, artistes, organisations syndicales et des professions, techniciens, administratifs, État, collectivité, financeurs, industrie etc. Tous ces experts (personnes, groupes, organisations) existent ! Ils sont bien réels et sont même pris en compte dans la définition des politiques mais, n'étant pas dotés de porte-paroles organisés ou reconnus, ils ne peuvent s'exprimer et faire valoir leurs intérêts. Logiquement, les experts patentés sont donc soupçonnés de détourner les systèmes à leur profit et la logique de la suspicion généralisée s'installe. La vraie réforme dont nous avons besoin consisterait à mettre au grand jour les réseaux qui constituent ce que l'on appelle la culture. À ce moment là, les combinazione se transformeront en arrangements démocratiques et en choix de sociétés ${ }^{10}$. Il est fort possible que les solutions résident moins dans des choix esthétiques (bien qu'il s'agisse aussi de ça) que d'inventer des procédures démocratiques qui permettent à la société de s'impliquer.

\section{- Exposer les désaccords et faire vite}

Même si l'affirmation peut sembler a priori paradoxale (ou gauchiste) dans la situation actuelle, la crise est en grande partie le résultat du consensus gauche-droite depuis 20 ans. En négligeant le débat démocratique, en excluant une série d'acteurs essentiels, sans cesse convoqués pour légitimer les politiques conduites (ou leur absence ou les tournants brutaux) et en concentrant les politiques sur les moyens et les équipements, c'est la politique culturelle que l'on a décrédibilisée. Le résultat, dont le dernier épisode consiste à débarquer manu militari des milliers de gens, sans même un plan social, est le triomphe de la logique comptable. D'autre part, le relativisme culturel a miné la "culture légitime ", sans pour autant que toutes les conséquences en soient tirées. Puisque nous savons que le goût évolue et qu'il n'existe pas de culture illégitime, le débat doit aussi se porter sur la définition des finalités des activités artistiques. Le fait qu'il soit revenu au MEDEF de prendre l'initiative en dit long sur l'inertie des animateurs du service public, sur l'immobilisme de l'État et sur le désarroi dans lequel nous vivons. Le tremblement de terre a au moins ouvert une brèche... 